\title{
Enhanced IgE allergic response to Aspergillus fumigatus in CFTR-I- mice
}

\begin{abstract}
Christian Müller ${ }^{1,2,3}$, Sofia A Braag ${ }^{1,3}$, John-David Herlihy ${ }^{2,4}$, Clive H Wasserfall ${ }^{3,5}$, Sarah E Chesrown ${ }^{1}$, Harry S Nick ${ }^{2,4}$, Mark A Atkinson ${ }^{3,5}$ and Terence R Flotte ${ }^{1,3}$

${ }^{1}$ Department of Pediatrics, Center for Immunology and Transplantation and Genetics Institute, University of Florida College of Medicine, Gainesville, FL, USA; ${ }^{2}$ Department of Molecular Genetics and Microbiology, Center for Immunology and Transplantation and Genetics Institute, University of Florida, Gainesville, FL, USA; ${ }^{3}$ Powell Gene Therapy Center, Center for Immunology and Transplantation and Genetics Institute, University of Florida, Gainesville, FL, USA; ${ }^{4}$ Department of Neurosciences, McKnight Brain Institute, Center for Immunology and Transplantation and Genetics Institute, University of Florida, Gainesville, FL, USA; and ${ }^{5}$ Department of Pathology, Center for Immunology and Transplantation and Genetics Institute, University of Florida, Gainesville, FL, USA
\end{abstract}

\begin{abstract}
To gain insight into aberrant cytokine regulation in cystic fibrosis (CF), we compared the phenotypic manifestations of allergen challenge in gut-corrected CFTR-deficient mice with background-matched C57BI6 (B6) mice. Aspergillus fumigatus (Af) antigen was used to mimic allergic bronchopulmonary aspergillosis, a peculiar hyper-IgE syndrome with a high prevalence in CF patients. CFTR-I-, C57BL/6 and FVB/NJ mice were sensitized with Af antigen by serial intraperitoneal injections. Control mice were mock sensitized with PBS. Challenges were performed by inhalation of Af antigen aerosol. After Af antigen challenge, histologic analysis showed goblet cell hyperplasia and lymphocytic infiltration in both strains. However, total serum IgE levels were markedly elevated in CF mice. Sensitized CF mice showed a five-fold greater IgE response to sensitization as compared with B6- and FVB-sensitized controls. Additional littermate controls to fully normalize for B6-FVB admixture in the strain background confirmed the role of CFTR mutation in the hyper-lgE syndrome. Cytokine mRNA levels of IL-5 and GM-CSF in the bronchoalveolar lavage (BAL) fluid, and BAL cell differentials indicated that CFTR mutation caused a shift from an IL-5-predominant to an IL-4-predominant cytokine profile. This system models a very specific type of airway inflammation in CF and could provide insights into pathogenesis and treatment of the disease.
\end{abstract}

Laboratory Investigation (2006) 86, 130-140. doi:10.1038/labinvest.3700379; published online 12 December 2005

Keywords: CFTR; allergy; inflammation; lung; IgE; Th1/Th2 cells

After the discovery of the CFTR gene 15 years ago, a detailed understanding of the pathogenic mechanisms whereby CFTR mutation leads to inflammatory lung disease remains elusive. Specific aberrations, which have been identified, include a deficiency of cAMP-activated chloride permeability, abnormal binding and internalization of Pseudomonas aeruginosa, and altered terminal glycosylation of surface and secreted glycoproteins. A growing body of evidence suggests that CFTR mutations also leads to an ERK kinase-mediated, ER overload response, which reprograms epithelial cell gene expression, which could predispose to an exaggerated proin-

Correspondence: Professor TR Flotte, MD, Department of Pediatrics, University of Florida College of Medicine, 1600 SW Archer Road, Box 100296, Gainesville, FL 32610-0296, USA.

E-mail: flotttr@peds.ufl.edu

Received 13 October 2005; revised 4 November 2005; accepted 15 November 2005; published online 12 December 2005 flammatory cytokine response in the lungs of cystic fibrosis (CF) patients. In addition, class I mutations that result in the production of nonfunctional CFTR protein have also been shown to cause aberrant inflammatory responses, characterized by increased levels of $\mathrm{TNF} \alpha, \mathrm{KC}$, mip2 and infective inflammatory responses toward Pseudomonas bacterial clearance. ${ }^{1}$ Ineffective and excessive inflammatory response to invading microbes in the $\mathrm{CF}$ airways seem to go beyond the effects of the CF mutation on the epithelial-bacterial interaction. This is evidenced by the data from CF infants, who despite having any detectable infection still produce elevated levels of proinflammatory cytokines. ${ }^{2}$ It is further supported by the similar findings in patients with mild pulmonary symptoms that show signs of excessive inflammation. . $^{3,4}$

This concept closely fits the progressive neutrophil-mediated airway damage seen in CF. The latter phenomenon has been recapitulated to some extent 
in the CFTR knockout mouse, although this model requires that agarose beads be used as a delivery vehicle for Pseudomonas in order to establish reproducible persistence of bacteria in the lower airways. Once this is established, however, there is a clear divergence between $\mathrm{CF}$ and normal mice, with CF mice demonstrating greater mortality, weight loss and lung inflammation. ${ }^{5}$

Another inflammatory phenotype that is quite peculiar to CF patients is a hyper-IgE syndrome known as allergic bronchopulmonary aspergillosis (ABPA), a syndrome characterized by an IgE antibody response specific for Aspergillus fumigatus (Af), a total serum IgE level of greater than $5000 \mathrm{ng} /$ $\mathrm{ml}$ and clinical signs of allergic airway hyperreactivity. ABPA is quite common in $\mathrm{CF}$ patients (10-15\% affected $)^{6,7}$ and very rare outside of CF. One report has indicated that even among those individuals who have ABPA without overt CF, approximately $50 \%$ will have one identifiable CFTR mutation. ${ }^{8}$ Animal model studies of ABPA have mainly focused on the use of C57BL/6 and Balb/c mice and respective cytokine knockouts in these genetic backgrounds. ${ }^{9-11}$ No animal studies have been conducted to mimic this peculiar allergic state in the genetic context of a CFTR knockout mouse. Despite the similarities in asthmatic ABPA and CF ABPA, the two also show differences in pathology and, more interestingly, in prevalence; this complicates the translation of the previous animal model results to $\mathrm{CF}$ and ABPA. Given the close connection between CFTR mutation and ABPA, we sought to determine whether CFTR knockout mice (CFTR S489X(-/-); fatty acid binding protein (FABP)hCFTR-gut corrected) ${ }^{12}$ were more prone to ABPA than normal mice, when sensitized with Af antigen and challenged via the airway. The observed hyperIgE phenotype could potentially serve as both a means to study pathogenic mechanism in CF lung disease and a model for studying the effect of new therapies in this disorder.

\section{Materials and methods}

\section{Mouse Strains}

The primary CFTR knockout strain used for these studies was the CFTR S489X(-/-) neo insertion in C57BL/6 mice developed initially at the University of North Carolina, ${ }^{13}$ and then modified with the transgenic overexpression of gut-specific expression of human CFTR from the FABP promoter in order to prevent intestinal obstruction and improve viability. ${ }^{12}$ These mice demonstrate the same lung phenotype as the original mice. ${ }^{5}$ Age- and sex-matched C57BL/6 mice were used as control mice for all experiments. Since some FVB/NJ genetic background remains in the CFTR S489X $(-/-)$; FABPhCFTR $(+/+)$ mice, FVB/NJ mice were also used as control mice.
In addition, CFTR S489X(-/-); FABP-hCFTR $(+/+)$ mice were backcrossed one more time to wild-type C57BL/6/mCFTR $(+/+)$ mice to produce CFTR S489X $(-/+)$; FABP-hCFTR $(+/+)$ mice. These mice were then crossed to generate F2 offspring littermates. The F2 littermates were genotyped, F2 CFTR S489X $(+/+)$; FABP-hCFTR $(+)$, F2 CFTR S489X $(-/+) ; \quad$ FABP-hCFTR $(+)$ and F2 CFTR S489X(-/-); FABP-hCFTR $(+)$, and were chosen for use in experiments analyzing IgE levels after sensitization and challenge with Af.

\section{Aspergillus Sensitization and Challenge}

CFTR S489X(-/-); FABP-hCFTR $(+/+)$, C57BL/6J and $\mathrm{FVB} / \mathrm{NJ}$ mice (5-6 weeks old) were housed in the SPF mouse colony of the University of Florida according to NIH guidelines and were allowed food and water ad libitum. All experimental procedures were approved by the IACUC of the University of Florida. Animals were sensitized to Af crude protein extract (Af-cpe) (Greer Laboratories). Briefly, animals were administered with intraperitoneal (i.p.) injections of $200 \mu \mathrm{g}$ of Af-cpe extract dissolved in $100 \mu \mathrm{l}$ of PBS on days 0 and 14 . Aerosol challenge was performed with $0.25 \%$ Af-cpe for $20 \mathrm{~min}$ in a $30 \times 30 \times 20 \mathrm{~cm}$ acrylic chamber using a jet nebulizer Pari model LC-D with an air flow of $6 \mathrm{l} / \mathrm{min}$ on days 28, 29 and 30. Nonsensitized control mice received i.p. injections with $\mathrm{PBS}$ alone and were challenged with Af-cpe along with sensitized mice.

\section{Serum IgE Levels}

Blood was collected on day 32 after killing the mice, centrifuged in a serum separator and stored at $-80^{\circ} \mathrm{C}$ until analysis. Total IgE levels were measured with the BD OptEIA ELISA (BD biosciences), and triplicate sera samples were used for each mouse. Tetramethyl-benzidine substrate was used to develop the assay and was read at $450 \mathrm{~nm}$ with correction at $570 \mathrm{~nm}$ on the VersaMax Micro Plate Reader (Molecular Devices).

Af antigen-specific ELISA was performed by coating a 96-well plates with $10 \mu \mathrm{g}$ of Af-cpe (Greer Laboratories) and detected using BD OptEIA IgE ELISA (BD biosciences) detection antibody. For the Af antigen-specific standard, the mouse serum from a high IgE responder was serially diluted starting at a 1:15 dilution. This relative standard was used to create a standard curve and its units were termed Ufs $/ \mathrm{ml}$, with the 1:15 dilution being $333 \mathrm{Ufs} / \mathrm{ml}$.

\section{Serum IgG Isotype Levels}

Blood was collected on day 32 after killing the mice, centrifuged in a serum separator and stored at 
$-80^{\circ} \mathrm{C}$ until analysis. Samples were assayed on the Bioplex Suspension Array system (Biorad) using the Beadlyte Mouse Immunoglobulin Isotyping Kit (Upstate) as per the manufacturer's instructions. Samples were diluted 1:25000.

\section{Bronchoalveolar Lavage (BAL) for Cell Counts and Cytokine Levels}

BAL fluid was retrieved from each animal via cannulation of the exposed trachea and gentle flushing of the lungs with two $1 \mathrm{ml}$ aliquots of PBS. Aliquots were pooled for individual animals preceding centrifugation and separation of pelleted cells and supernatant. Cytospin preparations of BAL cells were stained using Hema-3 (Biochemical Sciences, Swedesboro, NJ, USA) differential stain and relative cell populations were determined using standard morphological criteria.

\section{Cytokine Determination}

Assessments of cytokine profiles from the BAL were performed using a commercially available multiplexed kit (Biorad Mouse Multi-Cytokine Detection System; Biorad Laboratories) and the Bioplex Suspension Array System. Simultaneous measurement of five cytokines was performed: specifically IL- $1 \beta$, IL-2, IL-5, GM-CSF and IL-10. All assays were performed according to the manufacturer's protocols. Cytokine concentrations were determined utilizing Bioplex software with four-parameter data analysis. The sensitivity of the assay is less than $10 \mathrm{pg} / \mathrm{ml}$ and has a range from 0.2 to $32000 \mathrm{pg} / \mathrm{ml}$ with an inter- and intra-assay CV of less than $10 \%$.

\section{Lung Histology}

Immediately after collection of lavage fluid, one of the lobes was tied off, and ice-cold freshly prepared
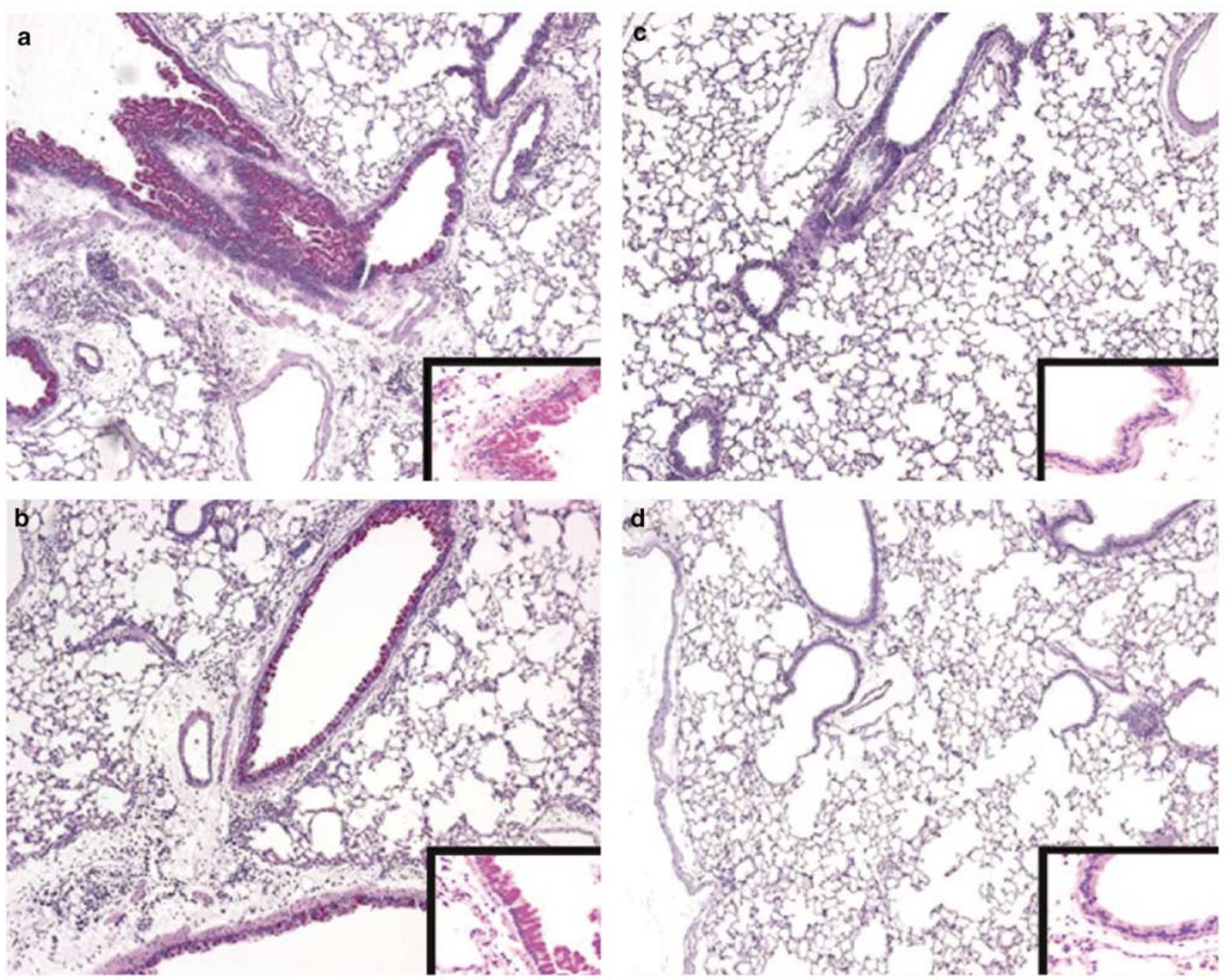

Figure 1 Representative PAS-stained whole-lung sections ( $\times 10)$ from $A$-challenged mice at day 32. C57BL/6 (a) and CFTR-/- (b) mice were sensitized with $200 \mu \mathrm{g}$ of $A f$ crude extract and C57BL/6 (c) and CFTR-/- (d) mice were PBS mock sensitized on days 1 and 14 . Inset pictures are $(\times 20)$ representative sections showing mucous cell hyperplasia. 
$4 \%$ paraformaldehyde in $1 \times$ PBS ( $\mathrm{pH}$ 7.4) was instilled through the tracheal cannula to one of the two lobes to inflation-fix the lung. Specimens were immersed in $4 \%$ paraformaldehyde at $4{ }^{\circ} \mathrm{C}$ overnight and dehydrated in a graded series of ethanol solutions. Tissue was embedded in paraffin. Sections were cut at $5 \mu \mathrm{m}$ thickness and mounted. For determination of inflammatory cells and goblet cell hyperplasia, PAS- and B220-stained slides were semiquantitatively scored in a blinded manner.

\section{Lung mRNA Extraction and Real-Time PCR}

RNA isolation, RT-PCR and real-time PCR-Total RNA was isolated from lung tissue as per the Chomczynski and Sacchi method with modifications. ${ }^{14}$ CDNA was produced from $1 \mu$ g of Total RNA using the Superscript First-Strand Synthesis System for RT-PCR (Invitrogen cat. \# 11904-018). The resultant cDNA was diluted 1:5 with sterile water for the subsequent real-time PCR. Real-time PCR was carried out in a $25 \mu \mathrm{l}$ reaction volume containing $2 \mu \mathrm{l}$ of diluted cDNA template, a final concentration of $1 \times$ SYBR Green Master Mix (ABI cat. \# 4309155) and $300 \mathrm{nM}$ each of forward and reverse primer. Reactions were carried out in ABI Prism 96well optical reaction plates with 96-well optical covers (Applied Biosystems). Amplification was conducted using an ABI Sequence Detection System 7000 (SDS 7000) and consisted of an initial hold for $2 \mathrm{~min}$ at $50^{\circ} \mathrm{C}, 10 \mathrm{~min}$ at $95^{\circ} \mathrm{C}$, followed by 40 cycles of melting at $95^{\circ} \mathrm{C}$ for $15 \mathrm{~s}$ and annealing/extension at $60^{\circ} \mathrm{C}$ for $1 \mathrm{~min}$. Amplification was determined automatically by the SDS 7000 instrument, and reported as a cycling threshold $\left(C_{\mathrm{T}}\right)$. Relative fold changes were determined using the $\Delta \Delta C_{\mathrm{T}}$ method, where the cyclophilin A gene has been used for normalization. ${ }^{15}$

\section{Statistical Analysis}

All data were compared using an unequal variance two-tailed Student's $t$-test, unless stated otherwise. Data were considered statistically significant when $P \leq 0.05$.

\section{Results}

\section{Sensitization-Specific Allergen-Induced Airway Inflammation}

In an attempt to model the peculiar airway inflammation phenotype of ABPA, CFTR-/ - and C57Bl/6, mice were sensitized and challenged with Af-cpe. At $48 \mathrm{~h}$ after the final aerosol Af-cpe challenge, lung tissue samples were obtained from the PBS- and Afsensitized mice to assess the degree of inflammatory cell infiltration and goblet cell hyperplasia. In order to control for nonspecific effects of Af antigen, we compared experimental animals with mice, which
Table 1 Analysis of lung tissue from CFTR-/- and C57BL/6 mice post-Af crude protein extract challenges

\begin{tabular}{lccc}
\hline & \multicolumn{3}{c}{ Peritracheal and peribronchial } \\
\cline { 2 - 4 } & Inflammation & Mucous HP & Edema \\
\hline CFTR-/- PBS & 0.0 & 0.0 & 0.0 \\
CFTR-/- Af & 0.0 & 2.0 & 1.0 \\
C57BL/6 PBS & 0.0 & 0.0 & 0.5 \\
C57BL/6 Af & 0.5 & 2.0 & 1.5
\end{tabular}

Perivascular

\begin{tabular}{lccc}
\cline { 2 - 4 } & Inflammation & Endothelitis & Edema \\
\hline CFTR-/- PBS & 0.5 & 0.0 & 1.0 \\
CFTR-/- Af & 2.0 & 2.0 & 2.0 \\
C57BL/6 PBS & 0.0 & 0.0 & 1.0 \\
C57BL/6 Af & 2.5 & 2.0 & 2.0
\end{tabular}

\begin{tabular}{lccc} 
& \multicolumn{3}{c}{ Alveolar and interstitial } \\
\cline { 2 - 4 } & Inflammation & Type II HP & Edema \\
\hline CFTR-/- PBS & 0.0 & 0.0 & 0.0 \\
CFTR-/- Af & 1.0 & 2.0 & 1.0 \\
C57BL/6 PBS & 0.0 & 0.0 & 0.0 \\
C57BL/6 Af & 1.5 & 1.5 & 1.5
\end{tabular}

PAS-stained slides from PBS- and Af-sensitized CFTR-/ - and C57Bl/ 6 mice were analyzed for the degree of infiltration around the airways and mucous cell hyperplasia. The following ranking system was used: $0=$ normal; $1=$ mild, focal; $2=$ mild, multifocal; $3=$ moderate, multifocal; 4 = severe, multifocal. Values are shown as the median value for a sample of $n=7$. Differences between Af- and PBS-sensitized animal are statistically significant $(P<0.05)$ using a Wilcoxon nonparametric $t$-test. There were no significant differences between Af-sensitized CFTR-/- and Af-sensitized C57BL/6.

have been mock sensitized with PBS injection and then challenged with the same dose of Af antigen. This latter group had no observable airway inflammation. In contrast, the Af-sensitized animals of both strains had a greater degree of mucous cell (Goblet cell) hyperplasia (Figure 1). Further histological analysis showed airway edema and alveolar inflammation only in Af-sensitized animals; however (Table 1), there were no demonstrable differences between strains in any of these indices, nor were there systematic strain differences in mast cell and B-cell (B220 staining) numbers (Figure 2).

\section{Hyper-IgE Response to Af in CF Mice}

The hallmark of ABPA in CF patients and the one parameter used for screening patients is the serum IgE level. In order to determine whether CF mice exhibited an analogous response to Af antigen, we compared serum IgE levels after Af-cpe sensitization and airway challenge with those seen in controls from comparable strain backgrounds and with other cohorts of unsensitized animals after acute Af antigen exposure (Figure 3). Total serum IgE levels 

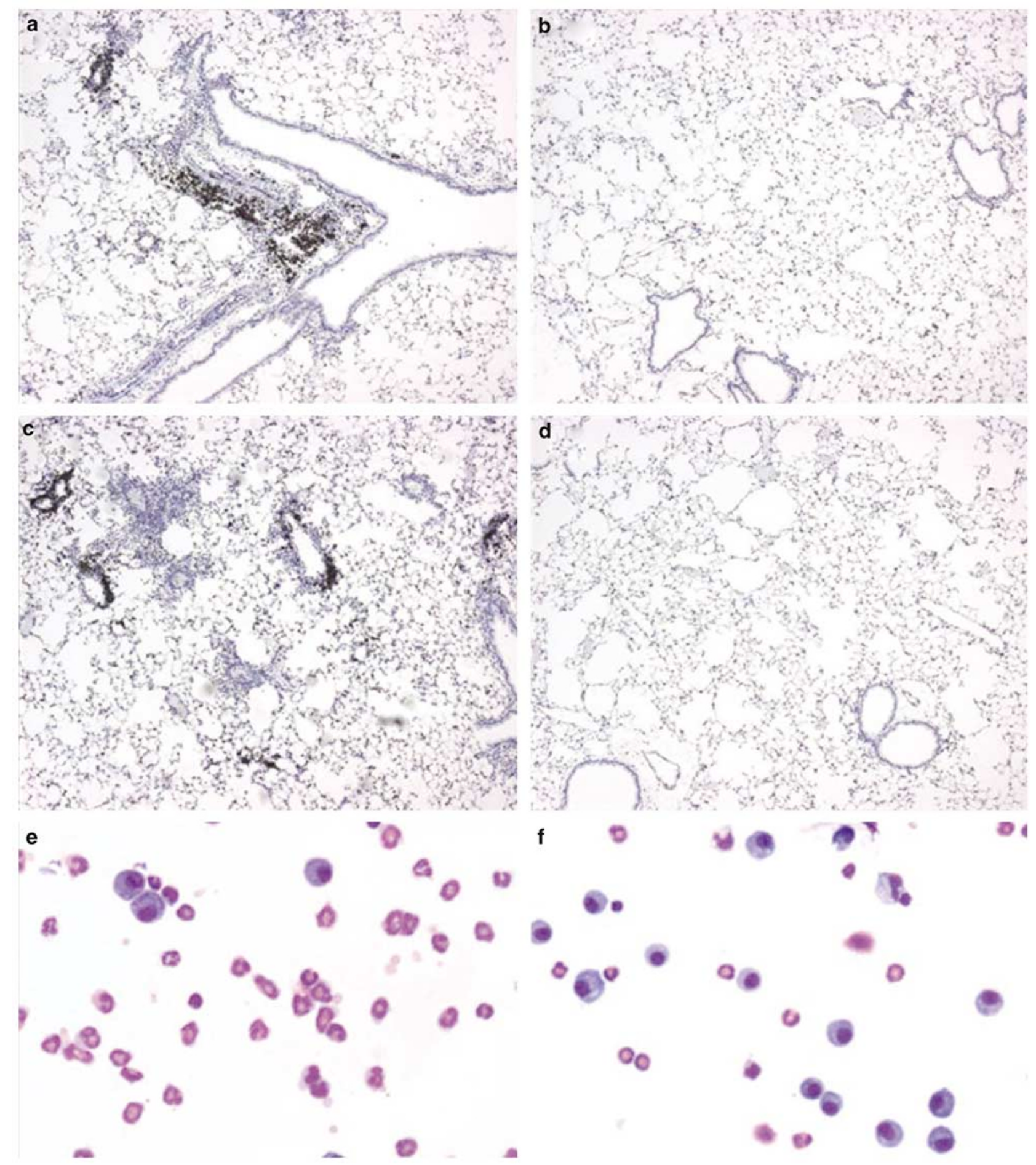

Figure 2 Representative B220-stained whole lung sections (a-d) from $A$ f-challenged mice and Hema-3-stained BAL infiltrates (e, f) at day 32. (a) CFTR-/- Af-sensitized, (b) CFTR-/- PBS-sensitized, (c) C57Bl/6 Af-sensitized and (d) C57Bl/6 PBS-sensitized mice. (e) Cytospin of the BAL from an Af-sensitized C57Bl/6. (f) Cytospin of the BAL from an Af-sensitized CFTR-/-. For quantitative analysis of BAL composition refer to Table 2.

in the Af-sensitized CF mice were extraordinarily high, reaching means of over $36000 \mathrm{ng} / \mathrm{ml}$, approximately fivefold greater than either the unsensitized CF controls or the sensitized non-CF controls. Interestingly, the 'baseline' levels of IgE in the CF mice (after the series of PBS 'sensitization' injections) were approximately equal to those seen in C57BL/6 mice, possibly indicating a predilection for IgE responsiveness in the CFTR-I- mice to environmental or nutritional factors even in the absence of 


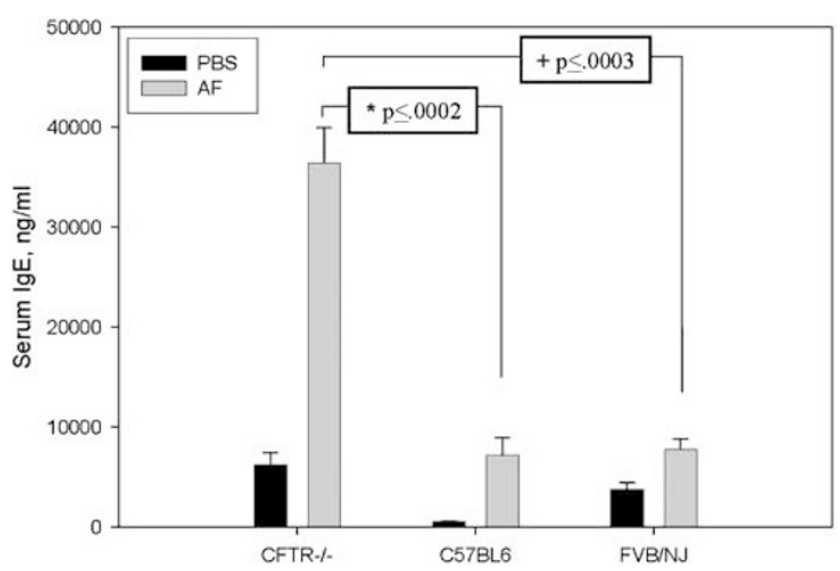

Figure 3 Total serum IgE levels in PBS-control or Af-sensitized CFTR-/ - (on a hybrid mostly C57BL/6, partial FVB background), C57BL/6 and FVB/NJ mice. CFTR $-/-(n=7), \mathrm{C} 57 \mathrm{BL} / 6(n=7)$ and FVB/NJ $(n=7)$ were sensitized with $200 \mu \mathrm{g}$ of Af crude extract dissolved in $200 \mu \mathrm{l}$ of PBS on days 1 and 14. Control mice for each strain $(n=7)$ were mock sensitized with $200 \mu$ l PBS. Blood samples were collected on day $32,48 \mathrm{~h}$ after the third aerosol Af challenge. Total serum IgE in the Af-sensitized $(\square)$ and PBScontrol sensitized ( $\mathbf{\square})$ mice were measured by ELISA. Mean \pm s.e.m. $(N=7)$.

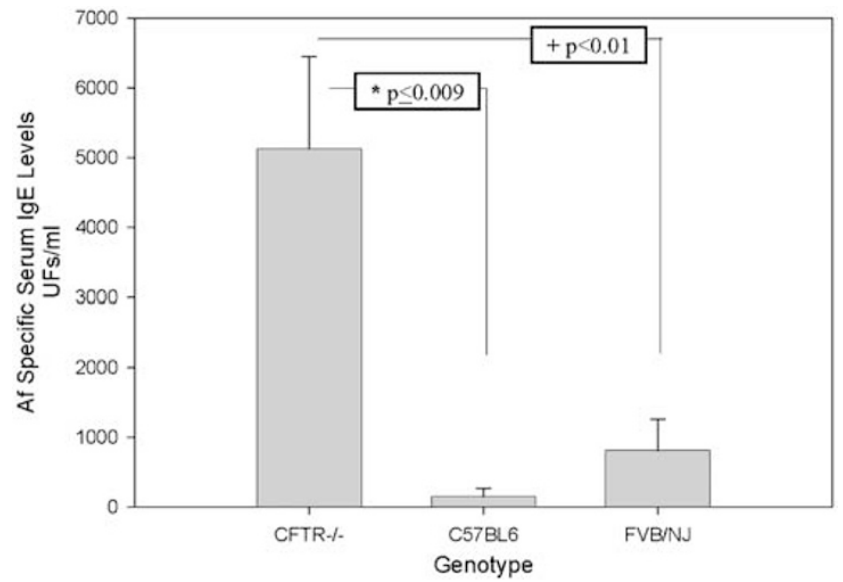

Figure 4 Af-specific serum IgE levels in Af-sensitized CFTR, C57BL/6 and FVB/NJ mice. CFTR $-/-(n=7)$, C57BL/6 $(n=7)$ and FVB/NJ $(n=7)$ were sensitized with $200 \mu \mathrm{g}$ of Af crude extract dissolved in $200 \mu \mathrm{l}$ of PBS on days 1 and 14. Serum IgE specific for Af crude extract was detected by coating the ELISA plate with $10 \mu \mathrm{g}$ of Af protein extract. Results are reported as Ufs $/ \mathrm{ml}$ (arbitrary units) created by serial dilution of a high IgE responder. Mean \pm s.e.m. $(N=7)$.

repeated Af sensitization. In agreement with the high total serum IgE levels, Af-sensitized mice also show an overwhelmingly higher Af antigen-specific IgE response. The levels of Af-specific IgE antibodies were again fivefold higher in CF mice than in FVB mice. Strikingly, the difference is even more pronounced (20-fold) when comparing CF mice to C57BL/6 (Figure 4).

Mouse strain background can have a profound effect on these responses, and the CF mouse strain used here is not on a pure C57BL/6 background.

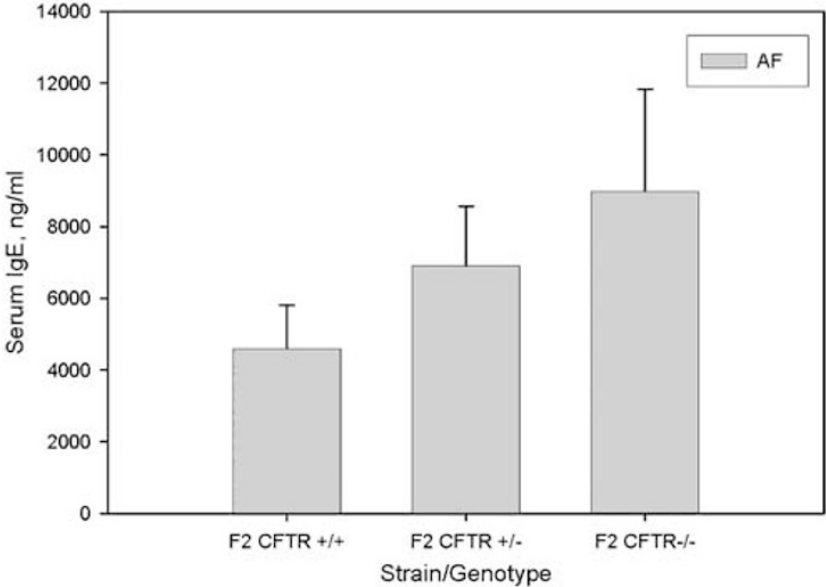

Figure 5 Total serum IgE levels in littermate controls. CFTR-/were backcrossed to $\mathrm{C} 57 \mathrm{BL} / 6$ to create $\mathrm{F} 1 \operatorname{mCFTR}(+/-)$ FABPhCFTR $(+)$. The F1 mice were bred to each other to create F2 hybrid background mice that were $\operatorname{mCFTR}(+/+), \operatorname{mCFTR}(+/-)$ and $\operatorname{mCFTR}(-/-)$. All mice were FABPhCFTR $(+)$, either homozygous or heterozygous for the latter transgene. All mice were sensitized with $200 \mu \mathrm{g}$ of Af crude extract on days 1 and 14 . Blood samples were collected on day $32,48 \mathrm{~h}$ after the third aerosol Af challenge. Total serum IgE was measured by ELISA. Results are reported as group means \pm s.e.m. $(N=8)$.

Therefore, we sought to further characterize the CFTR mutation's effects on total serum IgE levels by comparing mCFTR(-/-) mice with matched $\operatorname{mCFTR}(+/+)$ and heterozygous littermate controls. To accomplish this, we backcrossed the CFTR S489X(-/-); FABP-hCFTR $(+/+)$ mice to C57BL/ 6 , and then bred the resulting hybrid heterozygote offspring to each other to generate F2 hybrids. Litters from the $\mathrm{F} 2$ generation therefore included $\operatorname{mCFTR}(-/-), \operatorname{mCFTR}(+/-)$ and $\operatorname{mCFTR}(+/+)$ animals with the remainder of the background being of a consistent hybrid mixture. These experiments show a twofold increase in the levels of serum $\operatorname{IgE}$ levels from homozygous CFTR mutants when compared to mice homozygous for wild-type CFTR (Figure 5). The data also showed a strong trend toward a gene-dose response, with CFTR heterozygotes showing an intermediate level of total serum IgE when compared to $\operatorname{mCFTR}(+/+)$ and mCFTR(-/-) mice. It must be noted that all levels were significantly lower than those seen in the F0, which may indicate that strain background may play a partial role in determining total IgE levels as well.

To further characterize the hyperpolarized Th2 response, we looked at other antibody isotypes that respond to the effects of IL-4. Accordingly, the total serum IgG1 levels were twofold higher in the CFsensitized mice, providing evidence for an IL-4 hyperpolarized response in these mice consistent with clinical reports of CF-ABPA patients. Interestingly, IgG2b levels, which do not respond to IL-4 but rather to TGF- $\mathrm{B}^{16}$ and IgM levels were higher in nonCF controls (Figure 6). Finally, as seen in ABPA, IgG3 levels did not differ significantly among groups (data not shown). 
136
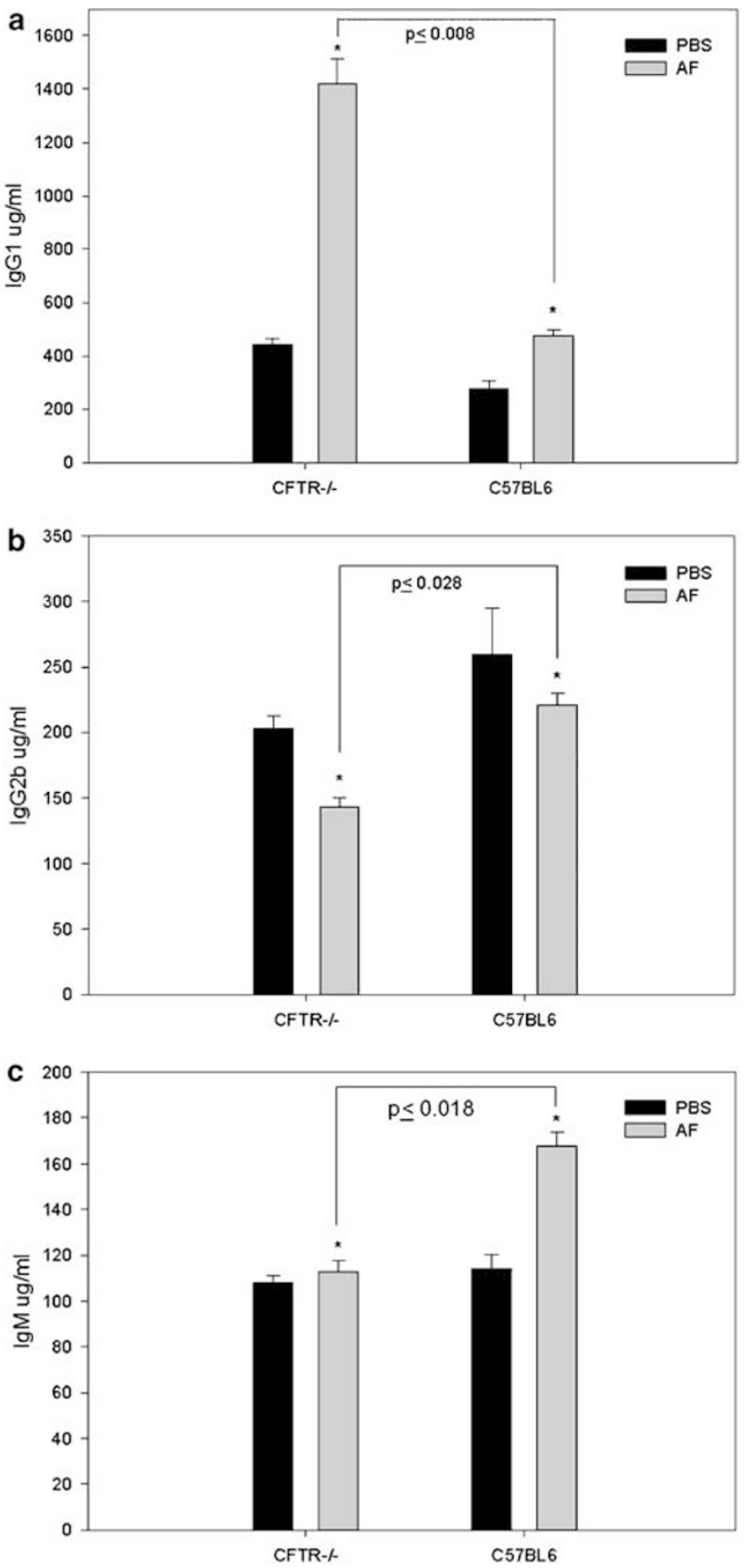

Figure 6 Total serum IgG subclass pattern in CF and non-CF mice sensitized to $A f$. Total serum (a) IgG1, (b) IgG2b and (c) IgM were measured in the Af-sensitized $(\square)$ and PBS-control sensitized (ם) mice using a multiplex bead assay on day $32,48 \mathrm{~h}$ after the last challenge. Mean \pm s.e.m. $(N=7)$.

\section{BAL Cell and Cytokine Profiles}

Mouse sensitization models of asthma generally follow either an IL-4-predominant or an IL-5predominant phenotype depending upon the strain background. ${ }^{17} \mathrm{~A}$ previous report indicated that the CFTR-/- mouse strain had upregulation of IL-4 expression, which also coincides with a high IgE level. ${ }^{18}$ In accordance with this initial finding in the CFTR mouse, recently Knutsen et $a l^{19}$ reported that ABPA CF patients have an increased sensitivity to IL-4 when compared to non-ABPA CF patients. This increased sensitivity resulted in a skewing of Th2T cells in ABPA CF patients. In order to test our working hypothesis that our CFTR-/ - Af-sensitized mice were exhibiting a similarly skewed IL-4-like response, we examined cell profiles and cytokine levels in the BAL of CFTR-/ - and control mice both at their baseline (PBS sensitized) and after Af sensitization (Table 2).

As demonstrated in Table 2, the CFTR-/- mice had a slight increase in BAL eosinophils at their baseline when compared to the C57BL/6 controls, and postsensitization exhibited a mixed cellular response. The C57BL/6 mice exhibited the expected macrophage predominance at baseline and a more completely eosinophilic response after Af sensitization and challenge. This pattern is completely consistent with the known predisposition of C57BL/6 mice to mount an IL-5-predominant response, while the CFTR-/- mice more closely mimicked the IL-4-predominant pathway.

Further direct confirmation of this phenotypic difference was provided by the measurement of BAL cytokine levels (Figure 7). As shown in Figure 6, the BAL IL-5 and BAL GM-CSF levels, two cytokines involved in the maturation recruitment and maintenance of eosinophils, ${ }^{20}$ were dramatically higher in the C57BL/6 mice than in the CFTR-/- mice, directly indicating an IL-5-predominant response in the former and an IL-4-biased response in the latter. Interestingly, IL-2 levels were also higher in the CFTR-/- mice, possibly indicating a more complex Th1/Th2 response. The dramatic differences in serum IgE levels, BAL cell count and BAL cytokine responses occurred despite ample histological evidence of airway and parenchymal inflammation in both strains argues for a qualitative different cytokine gene expression response rather than a simply quantitative difference in the degree of exposure and responsiveness.

\section{Cytokine Gene Expression Differences between CFTR-/- and Control Mice}

In order to confirm that phenotypic differences were due to the postulated differences in gene expression between the two strains, mRNA was analyzed from total lung extracts after Af-cpe challenge in all four groups (CFTR-/- Af sensitized, CFTR-/- PBS injected, C57BL/6 Af sensitized, C57BL/6 PBS injected) (Figure 8). As indicated in the figure, CFTR-/- mice demonstrated modest increases in IL-4 (twofold at baseline) and IL-2 (sevenfold after sensitization), and more dramatic increases in the chemokine KC (fourfold at baseline, 16-fold post-Afcpe challenge) and particularly in IL-13 (twofold at baseline, $>280$-fold post-Af). These changes 
Table 2 Proportions of inflammatory cells in the BAL

\begin{tabular}{lcccc}
\hline & C57BL/6 PBS (\%) & C57BL/6 Af (\%) & CFTR-/-PBS (\%) & CFTR-/-Af (\%) \\
\hline Macrophages & $96.6 \pm 1.0$ & $22.7 \pm 1.8^{*}$ & $93.6 \pm 2.9$ & $38.4 \pm 2.6^{*}$ \\
Lymphocytes & $1.7 \pm 0.4$ & $3.9 \pm 0.7$ & $2.1 \pm 0.8$ & $4.3 \pm 0.8$ \\
PMN & $0.2 \pm 0.1$ & $2.5 \pm 0.7$ & $1.3 \pm 0.6$ & $1.0 \pm 0.4$ \\
Eosinophils & $1.5 \pm 0.9$ & $71.2 \pm 2.3^{* *}$ & $3.7 \pm 2.1$ & $52.5 \pm 2.3^{* *}$ \\
\hline
\end{tabular}

Total BAL cellularity differential counts after aerosol challenge with Af crude extract. C57BL/6 and CFTR-/- mice were either PBS or Af sensitized and then challenged with $0.25 \%$ Af aerosol on 3 consecutive days (days 28-30). BAL cell counts were performed on day 32 . A measure of $100 \mu \mathrm{l}$ of BAL fluid was spun onto cytospin slides and no less than 300 cells were categorized per slide. Results are displayed as group average percentages of the total cells counted (errors are reported as s.e.m.; $N=7$ ).

${ }^{*} P \leq 0.00016$.

$* * P \leq 0.00019$.

parallel both the predilection for exaggerated IL-8like chemokine responses seen in human $\mathrm{CF}$ patients infected with P. aeruginosa and the IL-4/ IL-13-predominant asthma-like response that is seen in ABPA.

\section{Discussion}

The paucity of simple models of CF lung disease presents a limitation, both in studies of basic mechanisms of lung inflammation in $\mathrm{CF}$ and in studies of potential therapies. While a number of models of $P$. aeruginosa airway infection have been developed in CFTR knockout mice, each of these still presents some limitations.

In this report, we have taken an alternative approach, modeling a distinctly different inflammatory condition to which CF patients are uniquely susceptible. The observations presented here indicate that CFTR knockout mice do, indeed, demonstrate a qualitatively different response to Af antigen upon airway challenge, mimicking in many ways the hyper-IgE syndrome of ABPA.

The concept that cytokine gene regulation is aberrant in CF is far from novel. A range of studies dating back to the early 1980s have indicated that lung inflammation in $\mathrm{CF}$ may be exaggerated. ${ }^{21-24}$ Early studies indicated that anti-inflammatory corticosteroids may have benefits in CF lung disease generally, albeit with an unacceptable level of side effects, and steroid therapy remains standard of care in CF patients with ABPA. Other anti-inflammatory agents, such as ibuprofen, also have efficacy in CF lung disease. Studies from both CF cell lines and from the CFTR knockout mice indicate a predilection for higher levels of expression of certain proinflammatory cytokine genes either at baseline or after infection with Pseudomonas. A growing body of evidence also supports the notion that CFTR-related inflammation may actually precede infection. ${ }^{2}$ Recent studies suggest that the changes in inflammation caused by CF may be an inherent feature of immune cells involved in the inflammatory process as well as lung epithelial and submucosal gland serous cells. ${ }^{25-30}$ Finally, gene expression studies in the CFTR knockout mice have clearly indicated an upregulation of many genes, including IL- $4,{ }^{18}$ which likely forms the basis for the observations in the current study. In mice, total serum IgE and IgG1 are definitely correlated with IL4 production and a Th2-predominant response, so that our findings should have been predicted based on those studies.

The most striking feature of our model is the total IgE level itself, which is dramatically higher in Afsensitized CFTR knockout mice than in any other strain for which there is published data without the use of adjuvant. This feature in and of itself along with the Af-specific IgE levels mimics the clinical condition. In fact, a very large proportion of $\mathrm{CF}$ patients have anti-Af IgE and IgG antibodies, but only those with ABPA demonstrate total IgE levels greater than $10000 \mathrm{ng} / \mathrm{ml}$. The total serum IgE level, therefore, stands as the standard screening assay for this condition. In addition to the hyper-IgE response, Nikolaaizik et $a l^{31}$ has reported increased levels in IgG1 and IgG4 levels in CF ABPA patients when compared to non-ABPA CF controls. Thus, to further characterize the Th2 B-cell response, we looked at specific IgG isotypes. Whereas in humans, IgG4-like IgE is IL-4 responsive, in mice IL-4 drives the isotype switching of IgE and IgG1. ${ }^{32}$ Accordingly, accompanying the increase in IgE levels was the typical IL-4 antibody-driven response, which included higher levels of IgG1 antibodies in the sera of Af-sensitized CFTR knockout mice.

The exaggerated Th2-induced response that is observed in the CFTR knockout mice is probably driven by the higher basal levels of IL- 4 and IL-13 that were observed in our studies. The particularly robust and heightened induction of IL-13 in CF mice compared to the control mice is interesting, especially in the light of the recent report by Hauber et $a l,{ }^{33}$ in which he observes higher levels of IL-13 in the peripheral blood monocytes of CF patients. Interestingly, mRNA gene expression analysis also demonstrated a higher IL-2 and IFN-gamma response in the CFTR-/- Af-sensitized mice. Moreover, BAL samples from CF patients with ABPA demonstrate usually a mixed inflammatory pattern composed of a more complex Th1/Th2 lymphocyte 

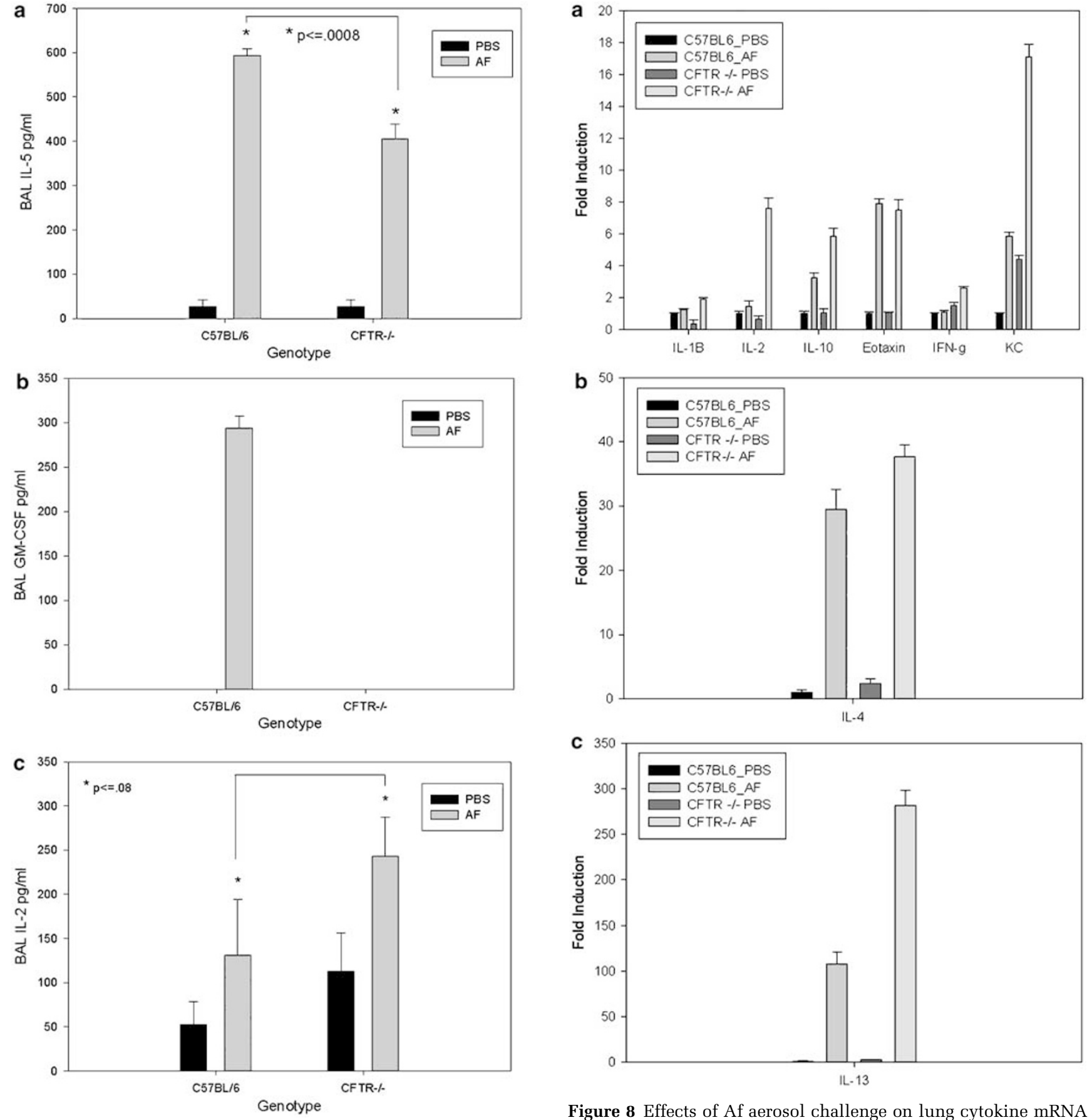

Figure 7 Effects of Af crude extract aerosol challenge on the cytokine levels in the BALs. (a) IL-5 BAL, (b) GM-CSF and (c) IL-2 BAL levels in PBS- $(\square)$ and Af- ( $\square)$ sensitized mice. Results are expressed as means \pm s.e.m. $(N=7)$.

response showing increased levels of IL-2 and IFNgamma along with IL-4 and IL-5. ${ }^{34}$ This mixed Th1/ Th2 response is more evident in the CFTR - / - mice showing higher levels of IL-2 and IFN-gamma along with IL-4 and IL-13 cytokines mimicking what is seen in the clinical setting. In addition to the upregulation of Th1/Th2 cytokines, there was also a fourfold increase in the levels of the chemokine

Figure 8 Effects of Af aerosol challenge on lung cytokine mRNA levels in PBS- and Af-sensitized mice. All mRNA levels are normalized as fold inductions over levels in the PBS-sensitized C57BL/6 mice. (a) Cytokines with less that 20-fold induction. (b) IL-4 and (c) IL-13 cytokine with highest induction levels. Relative fold changes were determined using the $\Delta \Delta C_{\mathrm{T}}$ method, where the cyclophilin A gene has been used for normalization. Data are represented as group means and error bars are s.d. $(N=7)$.

KC. KC is the murine counterpart of the human growth-related protein alpha (IL-8 family). The effects of KC in neutrophil trafficking and in response to Pseudomonas have been well documented. $^{5,35}$ Interestingly, this chemokine has been recently shown to induce a strong bronchial hyper- 
reactivity in an ovalbumin allergic mouse model via the upregulation of leukotrienes. ${ }^{36}$ In the same study, KC was also observed to act as a bridge between the innate and adaptive immune not only by serving as a chemoattractant but also by inducing the transcription of IL-13 mRNA in the lungs, which in turn amplifies the expression of $\mathrm{KC}$. This positive feedback regulation may help perpetuate and amplify the hyper-allergic phenomena observed in the CFTR knockout mice.

The murine model described here is unique in that the absence or dysfunction of the CFTR protein and its implications on the inflammatory response to inhaled allergen has not been described to date. While one might argue as to the relative importance of ABPA vs Pseudomonas infection in terms of global impact in this disease, the peculiar nature of this condition in CF suggests that it might provide some clues as to the cytokine aberrations that underlie both of these inflammatory lung phenotypes.

\section{Acknowledgements}

We thank Drs Tom Ferkol, David Strayer, and Mitchell Drumm for their helpful comments. This work was supported by grants from the NHLBI (HL51811), the CF Foundation and the Gold Coast CF Guild.

\section{References}

1 McMorran BJ, Palmer JS, Lunn DP, et al. G551D CF mice display an abnormal host response and have impaired clearance of Pseudomonas lung disease. Am J Physiol Lung Cell Mol Physiol 2001;281:L740-7.

2 Khan TZ, Wagener JS, Bost T, et al. Early pulmonary inflammation in infants with cystic fibrosis. Am J Respir Crit Care Med 1995;151:1075-1082.

3 Muhlebach MS, Stewart PW, Leigh MW, et al. Quantitation of inflammatory responses to bacteria in young cystic fibrosis and control patients. Am J Respir Crit Care Med 1999;160:186-191.

4 Noah TL, Black HR, Cheng PW, et al. Nasal and bronchoalveolar lavage fluid cytokines in early cystic fibrosis. J Infect Dis 1997;175:638-647.

5 Heeckeren A, Walenga R, Konstan MW, et al. Excessive inflammatory response of cystic fibrosis mice to bronchopulmonary infection with Pseudomonas aeruginosa. J Clin Invest 1997;100:2810-2815.

6 Laufer P, Fink JN, Bruns WT, et al. Allergic bronchopulmonary aspergillosis in cystic fibrosis. J Allergy Clin Immunol 1984;73:44-48.

7 Fink JN. Allergic bronchopulmonary aspergillosis. Hosp Pract (Off Ed) 1988;23:105-108 (13-4, 19-21 passim).

8 Miller PW, Hamosh A, Macek Jr M, et al. Cystic fibrosis transmembrane conductance regulator (CFTR) gene mutations in allergic bronchopulmonary aspergillosis. Am J Hum Genet 1996;59:45-51.
9 Kurup VP, Mauze S, Choi H, et al. A murine model of allergic bronchopulmonary aspergillosis with elevated eosinophils and IgE. J Immunol 1992;148: 3783-3788.

10 Hogaboam CM, Blease K, Schuh JM. Cytokines and chemokines in allergic bronchopulmonary aspergillosis (ABPA) and experimental Aspergillus-induced allergic airway or asthmatic disease. Front Biosci 2003; 8:e147-56.

11 Hogaboam CM, Gallinat CS, Taub DD, et al. Immunomodulatory role of $\mathrm{C} 10$ chemokine in a murine model of allergic bronchopulmonary aspergillosis. J Immunol 1999;162:6071-6079.

12 Zhou L, Dey CR, Wert SE, et al. Correction of lethal intestinal defect in a mouse model of cystic fibrosis by human CFTR. Science 1994;266:1705-1708.

13 Grubb BR, Pickles RJ, Ye $\mathrm{H}$, et al. Inefficient gene transfer by adenovirus vector to cystic fibrosis airway epithelia of mice and humans. Nature 1994;371: 802-806.

14 Visner GA, Dougall WC, Wilson JM, et al. Regulation of manganese superoxide dismutase by lipopolysaccharide, interleukin-1, and tumor necrosis factor. Role in the acute inflammatory response. J Biol Chem 1990; 265:2856-2864.

15 Livak KJ, Schmittgen TD. Analysis of relative gene expression data using real-time quantitative PCR and the 2(-Delta Delta C(T)) Method. Methods 2001; 25:402-408.

16 McIntyre TM, Klinman DR, Rothman P, et al. Transforming growth factor beta 1 selectivity stimulates immunoglobulin G2b secretion by lipopolysaccharideactivated murine B cells. J Exp Med 1993;177: 1031-1037.

17 Drazen JM, Arm JP, Austen KF. Sorting out the cytokines of asthma. J Exp Med 1996;183:1-5.

$18 \mathrm{Xu} \mathrm{Y,} \mathrm{Clark} \mathrm{JC,} \mathrm{Aronow} \mathrm{BJ,} \mathrm{et} \mathrm{al.} \mathrm{Transcriptional}$ adaptation to cystic fibrosis transmembrane conductance regulator deficiency. J Biol Chem 2003;278: 7674-7682.

19 Knutsen AP, Hutchinson PS, Albers GM, et al. Increased sensitivity to IL-4 in cystic fibrosis patients with allergic bronchopulmonary aspergillosis. Allergy 2004;59:81-87.

20 Miyajima A, Kinoshita $\mathrm{T}$, Wakao $\mathrm{H}$, et al. Signal transduction by the GM-CSF, IL-3 and IL-5 receptors. Leukemia 1997;11(Suppl 3):418-422.

21 Bonfield TL, Panuska JR, Konstan MW, et al. Inflammatory cytokines in cystic fibrosis lungs [published erratum appears in Am J Respir Crit Care Med 1996 Oct;154(4 Part 1):following 1217]. Am J Respir Crit Care Med 1995;152:2111-2118.

22 Chmiel JF, Konstan MW, Knesebeck JE, et al. IL-10 attenuates excessive inflammation in chronic Pseudomonas infection in mice. Am J Respir Crit Care Med 1999;160:2040-2047.

23 Konstan MW, Hilliard KA, Norvell TM, et al. Bronchoalveolar lavage findings in cystic fibrosis patients with stable, clinically mild lung disease suggest ongoing infection and inflammation [published erratum appears in Am J Respir Crit Care Med 1995 Jan;151(1):260]. Am J Respir Crit Care Med 1994; 150:448-454.

24 Konstan MW, Berger M. Current understanding of the inflammatory process in cystic fibrosis: onset and etiology. Pediatr Pulmonol 1997;24:137-142 (discussion 59-61). 
25 Tabary O, Zahm JM, Hinnrasky J, et al. Selective upregulation of chemokine IL-8 expression in cystic fibrosis bronchial gland cells in vivo and in vitro. Am J Pathol 1998;153:921-930.

26 Bonfield TL, Konstan MW, Berger M. Altered respiratory epithelial cell cytokine production in cystic fibrosis. J Allergy Clin Immunol 1999;104:72-78.

27 Jones MM, Seilheimer DK, Pier GB, et al. Increased elastase secretion by peripheral blood monocytes in cystic fibrosis patients. Clin Exp Immunol 1990;80: 344-349.

28 Pfeffer KD, Huecksteadt TP, Hoidal JR. Expression and regulation of tumor necrosis factor in macrophages from cystic fibrosis patients. Am J Respir Cell Mol Biol 1993;9:511-519.

29 Koller DY, Urbanek R, Gotz M. Increased degranulation of eosinophil and neutrophil granulocytes in cystic fibrosis. Am J Respir Crit Care Med 1995;152:629-633.

30 Kammouni W, Figarella C, Marchand S, et al. Altered cytokine production by cystic fibrosis tracheal gland serous cells. Infect Immun 1997;65:5176-5183.

31 Nikolaizik WH, Moser M, Crameri R, et al. Identification of allergic bronchopulmonary aspergillosis in cystic fibrosis patients by recombinant Aspergillus fumigatus I/a-specific serology. Am J Respir Crit Care Med 1995;152:634-639.

32 Deenick EK, Hasbold J, Hodgkin PD. Switching to IgG3, IgG2b, and IgA is division linked and independent, revealing a stochastic framework for describing differentiation. J Immunol 1999;163:4707-4714.

33 Hauber HP, Gholami D, Koppermann G, et al. Increased expression of Interleukin-13 but not interleukin-4 in cystic fibrosis patients. J Cyst Fibrosis 2003;2:189-194.

34 Walker C, Bauer W, Braun RK, et al. Activated T cells and cytokines in bronchoalveolar lavages from patients with various lung diseases associated with eosinophilia. Am J Respir Crit Care Med 1994;150:1038-1048.

35 Bozic CR, Kolakowski Jr LF, Gerard NP, et al. Expression and biologic characterization of the murine chemokine KC. J Immunol 1995;154:6048-6057.

36 Vargaftig BB, Singer M. Leukotrienes, IL-13, and chemokines cooperate to induce BHR and mucus in allergic mouse lungs. Am J Physiol Lung Cell Mol Physiol 2003;284:L260-L290. 